\title{
Series Solution for the Time-Fractional Coupled mKdV Equation Using the Homotopy Analysis Method
}

\author{
J. F. Gómez-Aguilar, ${ }^{1}$ H. Yépez-Martínez, ${ }^{2}$ R. F. Escobar-Jiménez, ${ }^{3}$ \\ V. H. Olivares-Peregrino, ${ }^{3}$ J. M. Reyes, ${ }^{2}$ and I. O. Sosa ${ }^{2}$ \\ ${ }^{1}$ CONACYT-Centro Nacional de Investigación y Desarrollo Tecnológico, Tecnológico Nacional de México, \\ Interior Internado Palmira S/N, Col. Palmira, 62490 Cuernavaca, MOR, Mexico \\ ${ }^{2}$ Universidad Autónoma de la Ciudad de México, Prolongación San Isidro 151, Col. San Lorenzo Tezonco, \\ Del. Iztapalapa, 09790 Ciudad de México, Mexico \\ ${ }^{3}$ Centro Nacional de Investigación y Desarrollo Tecnológico, Tecnológico Nacional de México, \\ Interior Internado Palmira S/N, Col. Palmira, 62490 Cuernavaca, MOR, Mexico
}

Correspondence should be addressed to J. F. Gómez-Aguilar; jgomez@ier.unam.mx

Received 22 June 2016; Accepted 8 September 2016

Academic Editor: Hang Xu

Copyright (C) 2016 J. F. Gómez-Aguilar et al. This is an open access article distributed under the Creative Commons Attribution License, which permits unrestricted use, distribution, and reproduction in any medium, provided the original work is properly cited.

We present new analytical approximated solutions for the space-time fractional nonlinear partial differential coupled mKdV equation. A homotopy analysis method is considered to obtain an infinite series solution. The effectiveness of this method is demonstrated by finding exact solutions of the fractional equation proposed, for the special case when the limit of the integral order of the time derivative is considered. The comparison shows a precise agreement between these solutions.

\section{Introduction}

Many important phenomena occurring in different fields of physics, chemistry, biology, signal processing, system identification, control theory, and finance dynamics and many other problems in different areas are frequently modeled through fractional differential equations [1-14]. Several methods have been developed to study the solutions of nonlinear fractional partial differential equations (NFPDEs), for instance, the variational iteration method [15-17], Adomian decomposition method [18-20], fractional subequation method [21-23], the homotopy perturbation technique [24$27]$, the homotopy analysis method (HAM) [28, 29], and Laplace homotopy analysis method $[30,31]$. However, for the nonlinear coupled equations with parameters derivative, especially fractional parameter derivative, not much work has been done [32].

A direct extension to the fractional-order case for the space-time fractional Hirota-Satsuma coupled $\mathrm{mKdV}$ equation takes the following form:

$$
\begin{aligned}
D_{t}^{\mu} u= & \frac{1}{2} D_{x}^{3 \mu} u-3 u^{2} D_{x}^{\mu} u+\frac{3}{2} D_{x}^{2 \mu} v+3 D_{x}^{\mu}(u v) \\
& -3 \lambda D_{x}^{\mu} u, \\
D_{t}^{\mu} v= & -D_{x}^{3 \mu} v-3 v D_{x}^{\mu} v-3\left(D_{x}^{\mu} u\right)\left(D_{x}^{\mu} v\right)+3 u^{2} D_{x}^{\mu} v \\
& +3 \lambda D_{x}^{\mu} v, \quad t>0,0<\mu \leq 1,
\end{aligned}
$$

where ${ }^{C} D_{x}^{\mu}$ and ${ }^{C} D_{t}^{\mu}$ are the Caputo derivatives [33], $\lambda$ is a constant, and $\mu$ is the order of the fractional derivative.

The aim of this work is to investigate the approximated analytical solutions of (1). The obtained solutions will be compared with the solutions obtained previously in the literature [34-37], for the coupled equation (1). The outline of this work is as follows: in Section 2, we describe the basic tools of fractional calculus. Section 3 contains the application of the method to obtain the approximated solutions for the coupled mKdV fractional system. In Section 4, we compared 
our results with those reported in the literature [38]. Finally in Section 5 conclusions are given.

\section{Fractional Calculus}

The Riemann-Liouville operator is defined as $[39,40]$

$$
{ }^{\mathrm{RL}} D^{\mu} f(x)=\frac{1}{\Gamma(\mu)} \int_{0}^{x}(x-t)^{\mu-1} f(t) d t, \quad \mu>0 .
$$

The fractional derivative of $f(x)$ in the Caputo sense is defined as

$$
\begin{aligned}
{ }^{C} D^{\mu} f(x) & ={ }^{\mathrm{RL}} D^{n-\mu} \frac{d^{n}}{d x^{n}}[f(x)] \\
& =\frac{1}{\Gamma(n-\mu)} \int_{0}^{x} \frac{f^{(n)}(t)}{(x-t)^{\mu-n+1}} d t,
\end{aligned}
$$

for $n-1<\mu \leq n, n \in N, x>0, f \in \mathbb{C}^{n}$.

The fractional derivative of $f(x)$ in the Caputo sense satisfies the following relations:

$$
\begin{aligned}
& { }^{\mathrm{RL}} D^{\mu \mathrm{C}} D^{\mu} f(x)=f(x)-\sum_{k=0}^{n-1} f^{(n)}\left(0^{+}\right) \frac{x^{k}}{k !}, \quad x>0, \\
& { }^{C} D^{\mu \mathrm{RL}} D^{\mu} f(x)=f(x) .
\end{aligned}
$$

The Caputo operator is used here because the initial conditions for the fractional differential equations can be handled by using an analogy with the classical case (ordinary derivative).

\section{The HAM Applied to Coupled mKdV Equation}

Consider the nonlinear space-time fractional coupled $\mathrm{mKdV}$ equation

$$
\begin{aligned}
{ }^{C} D_{t}^{\mu} u= & \frac{1}{2}{ }^{C} D_{x}^{3 \mu} u-3 u^{2 C} D_{x}^{\mu} u+\frac{3}{2}{ }^{C} D_{x}^{2 \mu} v+3{ }^{C} D_{x}^{\mu}(u v) \\
& -3 \lambda^{C} D_{x}^{\mu} u, \\
{ }^{C} D_{t}^{\mu} v= & -{ }^{C} D_{x}^{3 \mu} v-3 v^{C} D_{x}^{\mu} v-3\left({ }^{C} D_{x}^{\mu} u\right)\left({ }^{C} D_{x}^{\mu} v\right) \\
& +3 u^{2 C} D_{x}^{\mu} v+3 \lambda^{C} D_{x}^{\mu} v,
\end{aligned}
$$

$$
t>0,0<\mu \leq 1
$$

with the initial conditions

$$
\begin{aligned}
& u(x, t)=\frac{b^{\mu}}{2 k^{\mu}}+k^{\mu} \tanh _{\mu}(k x), \\
& v(x, t)=\lambda+b^{\mu} \tanh _{\mu}(k x) .
\end{aligned}
$$

The exact solutions obtained by applying the fractional subequation method are given by $[41,42]$

$$
\begin{aligned}
& u(x, t)=\frac{b^{\mu}}{2 k^{\mu}}+k^{\mu} \tanh _{\mu}(k x+c t), \\
& v(x, t)=\lambda+b^{\mu} \tanh _{\mu}(k x+c t),
\end{aligned}
$$

where

$$
c^{\mu}=-k^{3 \mu}+\frac{3}{4} k^{\mu}\left(\frac{b^{\mu}}{k^{\mu}}\right)^{2}
$$

By means of HAM, we choose the linear operator [43]

$$
\mathscr{L}[\Theta(x, t ; p)]={ }^{C} D_{t}^{\mu} \Theta(x, t ; p),
$$

with $\mathscr{L}[d]=0$ and $\Theta(x, t ; p)=u(x, t ; p)=u$ or $\Theta(x, t ; p)=v(x, t ; p)=v$. We define the nonlinear operator $\mathcal{N}_{i}\left[{ }^{C} D^{\mu} \Theta(x, t ; p)\right], i=0,1,2,3, \ldots, n$, as

$$
\begin{aligned}
& \mathcal{N}_{1}\left[u,{ }^{C} D^{\mu} u, v,{ }^{C} D^{\mu} v\right]={ }^{C} D_{t}^{\mu} u-\left(\frac{{ }^{1}}{2}{ }^{C} D_{x}^{3 \mu} u\right. \\
& \left.-3 u^{2 C} D_{x}^{\mu} u+\frac{3}{2}{ }^{C} D_{x}^{2 \mu} v+3{ }^{C} D_{x}^{\mu} u v-3 \lambda^{C} D_{x}^{\mu}\right), \\
& \mathcal{N}_{2}\left[u,{ }^{C} D^{\mu} u, v,{ }^{C} D^{\mu} v\right]={ }^{C} D_{t}^{\mu} v-\left(-{ }^{C} D_{x}^{3 \mu} v-3 v^{C} D_{x}^{\mu} v\right. \\
& \left.-3\left({ }^{C} D_{x}^{\mu} u\right)\left({ }^{C} D_{x}^{\mu} v\right)+3 u^{2 C} D_{x}^{\mu} v+3 \lambda^{C} D_{x}^{\mu} v\right) .
\end{aligned}
$$

We construct the zero-order deformation equation

$$
\begin{aligned}
& (1-p) \mathscr{L}\left(u-u_{0}\right) \\
& \quad=p H(t) h \mathcal{N}_{1}\left[u,{ }^{C} D^{\mu} u, v,{ }^{C} D^{\mu} v\right], \\
& (1-p) \mathscr{L}\left(v-v_{0}\right)=p H(t) h \mathcal{N}_{2}\left[u,{ }^{C} D^{\mu} u, v,{ }^{C} D^{\mu} v\right],
\end{aligned}
$$

where $h$ is a nonzero auxiliary parameter; when $p=0$ and $p=1$, we have [44]

$$
\begin{aligned}
& \Theta_{i}(x, t ; 0)=u_{i 0}(x, t) \\
& \Theta_{i}(x, t ; 1)=u_{i}(x, t) .
\end{aligned}
$$

Now we obtain the $m$ th order deformation equation

$$
\begin{aligned}
& \mathscr{L}\left(u_{m}-\kappa_{m} u_{m-1}\right)=h H(t) R_{m}^{(1)}\left(\mathbf{u}_{m-1}, \mathbf{v}_{m-1}\right), \\
& \mathscr{L}\left(v_{m}-\kappa_{m} v_{m-1}\right)=h H(t) R_{m}^{(2)}\left(\mathbf{u}_{m-1}, \mathbf{v}_{m-1}\right),
\end{aligned}
$$

where

$$
\begin{aligned}
& R_{m}^{(1)}\left(\mathbf{u}_{m-1}, \mathbf{v}_{m-1}\right)={ }^{C} D_{t}^{\mu} u_{m-1}-\left[\frac{1}{2}{ }^{C} D_{x}^{3 \mu} u_{m-1}\right. \\
& \quad-3\left(\sum_{i=0}^{m-1} \sum_{j=0}^{i} u_{i-j} u_{j}{ }^{C} D_{x}^{\mu} u_{m-1-i}\right)+\frac{3}{2}{ }^{C} D_{x}^{2 \mu} v_{m-1}
\end{aligned}
$$




$$
\begin{aligned}
& -3 \sum_{i=0}^{m-1}\left(u_{i}^{C} D_{x}^{\mu} v_{m-1-i}+v_{i}{ }^{C} D_{x}^{\mu} u_{m-1-i}\right) \\
& \left.-3 \lambda^{C} D_{x}^{\mu} u_{m-1}\right] \\
& R_{m}^{(2)}\left(\mathbf{u}_{m-1}, \mathbf{v}_{m-1}\right)={ }^{C} D_{t}^{\mu} v_{m-1}-\left[-{ }^{C} D_{x}^{3 \mu} v_{m-1}\right. \\
& -3 \sum_{i=0}^{m-1}\left(v_{m-1-i}{ }^{C} D_{x}^{\mu} v_{i}+\left({ }^{C} D_{x}^{\mu} v_{i}\right)\left({ }^{C} D_{x}^{\mu} u_{m-1-i}\right)\right) \\
& \left.+3\left(\sum_{i=0}^{m-1} \sum_{j=0}^{i} u_{i-j} u_{j}{ }^{C} D_{x}^{\mu} v_{m-1-i}\right)+3 \lambda^{C} D_{x}^{\mu} v_{m-1}\right] .
\end{aligned}
$$

For the auxiliary function $H(t)$, we can take $H(t)=1$ $[44,45]$. Applying the Riemann-Liouville operator ${ }^{\mathrm{RL}} D^{\mu}$, the solution of the $m$ th order deformation equation (14) for $m \geq 1$ becomes

$$
\begin{aligned}
& u_{m}=\kappa_{m} u_{m-1}+h^{\mathrm{RL}} D^{\mu} R_{m}^{(1)}\left(\mathbf{u}_{m-1}, \mathbf{v}_{m-1}\right), \\
& v_{m}=\kappa_{m} v_{m-1}+h^{\mathrm{RL}} D^{\mu} R_{m}^{(2)}\left(\mathbf{u}_{m-1}, \mathbf{v}_{m-1}\right) .
\end{aligned}
$$

Now if we substitute the initial condition (6), system (15) takes the following form:

$$
\begin{aligned}
& p^{0}: u_{0}(x, 0)=\frac{b^{\mu}}{2 k^{\mu}}+k^{\mu} \tanh _{\mu}(k x+c t) \\
& v_{0}(x, 0)=\lambda+b^{\mu} \tanh _{\mu}(k x+c t) \\
& p^{1}: u_{1}(x, t)=h^{\mathrm{RL}} D^{\mu}\left\{-\left[\frac{1}{2}{ }^{C} D_{x}^{3 \mu} u_{0}-3 u_{0}^{2 C} D_{x}^{\mu} u_{0}\right.\right. \\
& \quad+\frac{3}{2}{ }^{C} D_{x}^{2 \mu} v_{0}+3 u_{0}{ }^{C} D_{x}^{\mu} v_{0}+3 v_{0}{ }^{C} D_{x}^{\mu} u_{0} \\
& \left.\left.\quad-3 \lambda^{C} D_{x}^{\mu} u_{0}\right]\right\}, \\
& u_{1}(x, 0)=0, \\
& v_{1}(x, t)=h^{\mathrm{RL}} D^{\mu}\left\{-\left[-{ }^{C} D_{x}^{3 \mu} v_{0}-3 v_{0}{ }^{C} D_{x}^{\mu} v_{0}\right.\right. \\
& \left.\left.\quad-3\left({ }^{C} D_{x}^{\mu} u_{0}\right)\left({ }^{C} D_{x}^{\mu} v_{0}\right)+3 u_{0}^{2 C} D_{x}^{\mu} v_{0}+3 \lambda^{C} D_{x}^{\mu} v_{0}\right]\right\} \\
& v_{1}(x, 0)=0, \\
& p^{2}: u_{2}(x, t)-u_{1}(x, t)=h^{\mathrm{RL}} D^{\mu}\left\{{ }^{C} D_{t}^{\mu} u_{1}\right. \\
& \quad-\left[\frac{1}{2}{ }^{C} D_{x}^{3 \mu} u_{1}-3 u_{0}^{2 C} D_{x}^{\mu} u_{1}-6 u_{1} u_{0}{ }^{C} D_{x}^{\mu} u_{0}\right.
\end{aligned}
$$

$$
\begin{aligned}
& +\frac{3}{2}{ }^{C} D_{x}^{2 \mu} v_{1}+3 u_{0}{ }^{C} D_{x}^{\mu} v_{1}+3 v_{1}{ }^{C} D_{x}^{\mu} u_{0}+3 v_{0}{ }^{C} D_{x}^{\mu} u_{1} \\
& \left.\left.+3 u_{1}{ }^{C} D_{x}^{\mu} v_{0}-3 \lambda^{C} D_{x}^{\mu} u_{1}\right]\right\} \\
& u_{2}(x, 0)=0 \\
& v_{2}(x, t)-v_{1}(x, t)=h^{\mathrm{RL}} D^{\mu}\left\{{ }^{C} D_{t}^{\mu} v_{1}-\left[-{ }^{C} D_{x}^{3 \mu} v_{1}\right.\right. \\
& -3 v_{1}{ }^{C} D_{x}^{\mu} v_{0}-3 v_{0}{ }^{C} D_{x}^{\mu} v_{1}-3\left({ }^{C} D_{x}^{\mu} u_{0}\right)\left({ }^{C} D_{x}^{\mu} v_{1}\right) \\
& -3\left({ }^{C} D_{x}^{\mu} u_{1}\right)\left({ }^{C} D_{x}^{\mu} v_{0}\right)+3 u_{0}^{2 C} D_{x}^{\mu} v_{1}+6 u_{1} u_{0}{ }^{C} D_{x}^{\mu} v_{0} \\
& \left.\left.+3 \lambda^{C} D_{x}^{\mu} v_{1}\right]\right\} \text {, } \\
& v_{2}(x, 0)=0 \text {, } \\
& p^{3}: u_{3}(x, t)-u_{2}(x, t)=h^{\mathrm{RL}} D^{\mu}\left\{{ }^{C} D_{t}^{\mu} u_{2}\right. \\
& -\left[\frac{1}{2}{ }^{C} D_{x}^{3 \mu} u_{2}-3 u_{0}^{2 C} D_{x}^{\mu} u_{2}-6 u_{2} u_{0}{ }^{C} D_{x}^{\mu} u_{0}\right. \\
& -6 u_{1} u_{0}{ }^{C} D_{x}^{\mu} u_{1}-3 u_{1}^{2 C} D_{x}^{\mu} u_{0}+\frac{3}{2}{ }^{C} D_{x}^{2 \mu} v_{2} \\
& +3 u_{0}{ }^{C} D_{x}^{\mu} v_{2}+3 v_{2}{ }^{C} D_{x}^{\mu} u_{0}+3 v_{1}{ }^{C} D_{x}^{\mu} u_{1}+3 v_{0}{ }^{C} D_{x}^{\mu} u_{2} \\
& \left.\left.+3 u_{2}{ }^{C} D_{x}^{\mu} v_{0}+3 u_{1}{ }^{C} D_{x}^{\mu} v_{1}-3 \lambda^{C} D_{x}^{\mu} u_{2}\right]\right\} \text {, } \\
& u_{3}(x, 0)=0 \text {, } \\
& v_{3}(x, t)-v_{2}(x, t)=h^{\mathrm{RL}} D^{\mu}\left\{{ }^{C} D_{t}^{\mu} v_{2}-\left[-{ }^{C} D_{x}^{3 \mu} v_{2}\right.\right. \\
& -3 v_{2}{ }^{C} D_{x}^{\mu} v_{0}-3 v_{1}{ }^{C} D_{x}^{\mu} v_{1}-3 v_{0}{ }^{C} D_{x}^{\mu} v_{2} \\
& -3\left({ }^{C} D_{x}^{\mu} u_{0}\right)\left({ }^{C} D_{x}^{\mu} v_{2}\right)-3\left({ }^{C} D_{x}^{\mu} u_{1}\right)\left({ }^{C} D_{x}^{\mu} v_{1}\right) \\
& -3\left({ }^{C} D_{x}^{\mu} u_{2}\right)\left({ }^{C} D_{x}^{\mu} v_{0}\right)+3 u_{0}^{2 C} D_{x}^{\mu} v_{2}+6 u_{2} u_{0}{ }^{C} D_{x}^{\mu} v_{0} \\
& \left.\left.+6 u_{1} u_{0}{ }^{C} D_{x}^{\mu} v_{1}+3 u_{1}^{2 C} D_{x}^{\mu} v_{0}+3 \lambda^{C} D_{x}^{\mu} v_{2}\right]\right\} \text {, } \\
& v_{3}(x, 0)=0 \text {, }
\end{aligned}
$$

Solving with Mathematica software, the approximate solutions of (5), considering $h=-1$ [44], are

$$
\begin{aligned}
& p^{0}: u_{0}(x, 0)=\frac{b^{\mu}}{2 k^{\mu}}+k^{\mu} \tanh _{\mu}(k x+c t), \\
& v_{0}(x, 0)=\lambda+b^{\mu} \tanh _{\mu}(k x+c t), \\
& p^{1}: u_{1}(x, t)=\frac{t^{\mu}\left(4 k^{4 \mu}-3 b^{2 \mu}\right)\left(-1+\tanh _{\mu}(k x)^{2}\right)}{4 \Gamma(\mu+1)}, \\
& v_{1}(x, t)=\frac{t^{\mu}(b k)^{\mu}\left(4 k^{2 \mu}-3(b / k)^{2 \mu}\right)\left(-1+\tanh _{\mu}(k x)^{2}\right)}{4 \Gamma(\mu+1)},
\end{aligned}
$$




$$
\begin{aligned}
p^{2}: & u_{2}(x, t) \\
& =\frac{\left(3 b^{2 \mu}-4 k^{4 \mu}\right)^{2}\left(t^{2} / k\right)^{\mu} \tanh _{\mu}(k x)\left(-1+\tanh _{\mu}(k x)^{2}\right)}{8 \Gamma(2 \mu+1)},
\end{aligned}
$$$$
v_{2}(x, t)
$$

$$
=\frac{b^{\mu}\left(3 b^{2 \mu}-4 k^{4 \mu}\right)^{2}(t / k)^{2 \mu} \tanh _{\mu}(k x)\left(-1+\tanh _{\mu}(k x)^{2}\right)}{8 \Gamma(2 \mu+1)},
$$

\section{Discussion}

Next, we will compare the approximated analytical solution obtained in the above section with the exact analytical solution previously obtained in [41]. In this comparison, using Mathematica, setting $p=1$ in the solutions obtained by the HAM to the coupled mKdV system eq. (17), we can write for $\mu=1$ (classical case)

$$
\begin{aligned}
& u(x, t)=\frac{b}{2 k}+k \tanh (k x)+\frac{1}{4} t\left(3 b^{2}-4 k^{4}\right) \operatorname{sech}^{2}(k x) \\
& -\frac{t^{2}\left(3 b^{2}-4 k^{4}\right)^{2} \tanh (k x) \operatorname{sech}^{2}(k x)}{16 k} \\
& +\frac{t^{3}\left(3 b^{2}-4 k^{4}\right)^{3}(\cosh (2 k x)-2) \operatorname{sech}^{4}(k x)}{192 k^{2}} \\
& -\frac{t^{4}\left(3 b^{2}-4 k^{4}\right)^{4}(\cosh (2 k x)-5) \tanh (k x) \operatorname{sech}^{4}(k x)}{1536 k^{3}} \\
& +\cdots=\frac{b}{2 k}+k \tanh \left(k x+\left(-k^{3}+\frac{3 b^{2}}{4 k}\right) t\right), \\
& v(x, t)=\lambda+b \tanh (k x)+\frac{b t\left(3 b^{2}-4 k^{4}\right) \operatorname{sech}^{2}(k x)}{4 k} \\
& -\frac{b t^{2}\left(3 b^{2}-4 k^{4}\right)^{2} \tanh (k x) \operatorname{sech}^{2}(k x)}{16 k^{2}} \\
& +\frac{b t^{3}\left(3 b^{2}-4 k^{4}\right)^{3}(\cosh (2 k x)-2) \operatorname{sech}^{4}(k x)}{192 k^{3}} \\
& -\frac{b t^{4}\left(3 b^{2}-4 k^{4}\right)^{4}(\cosh (2 k x)-5) \tanh (k x) \operatorname{sech}^{4}(k x)}{1536 k^{4}} \\
& +\cdots=\lambda+b \tanh \left(k x+\left(-k^{3}+\frac{3 b^{2}}{4 k}\right) t\right),
\end{aligned}
$$

which is the exact solution when $\mu=1$, in the general solutions (7), that has been obtained previously, by applying the fractional subequation method [41]. Similar results have been obtained for the nonlinear fractional-order coupled $\mathrm{mKdV}$ equation when only time-fractional dependence has been considered in the HAM [36], but with integer order

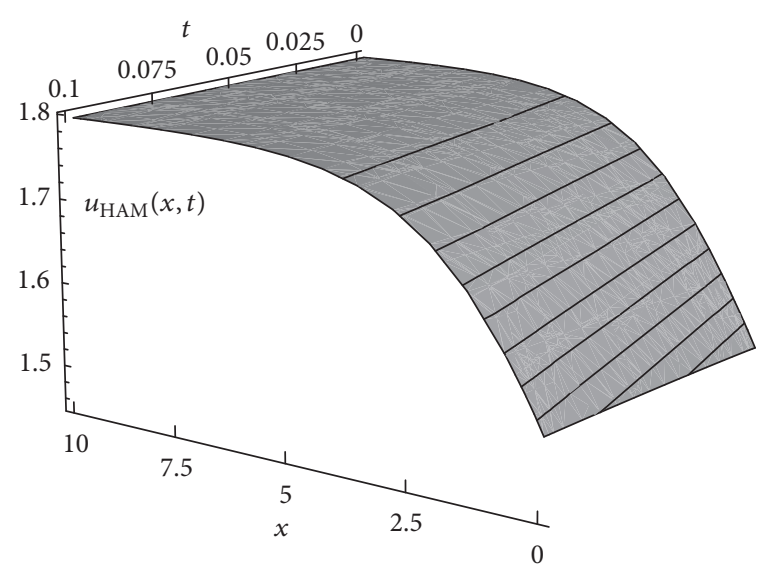

FIGURE 1: Approximated analytical solutions eq. (17) for $u(x, t)$, with $\lambda=0.1, b=1 ; k=1 / 3$, at $t=0.001$ and $\mu=0.97$.

derivatives for space variables. However, in [36], they have compared their results with the exact analytical results obtained by Fan [38], for the classical case $\mu=1$, that is,

$$
\begin{aligned}
& \tilde{u}(x, t)=\frac{b}{2 k}+k \tanh (k x+\tilde{c} t), \\
& \widetilde{v}(x, t)=\frac{\lambda}{2}\left(1+\frac{k}{b}\right)+b \tanh (k x+\widetilde{c} t),
\end{aligned}
$$

where in the classical solution of Fan the following dispersion relation was obtained [38]:

$$
\widetilde{c}=-k^{3}+\frac{3}{4} k\left(\frac{b}{k}\right)^{2}+\frac{6}{4} k \lambda\left(\frac{k}{b}-1\right) .
$$

Figures 1 and 2 show the numerical solutions of the nonlinear coupled $\mathrm{mKdV}$ equations with $\lambda=0.1, b=1 ; k=$ $1 / 3$, at $t=0.001$ and $\mu=0.97$. Figures 3 and 4 depict the exact solutions (7), with $\lambda=0.1, b=1, k=1 / 3, t=0.001$, and $\mu=0.97$.

Figures 5 and 6 show the absolute difference between the exact solutions (7) and the approximated solutions (17), where we have taken into account the first five terms in the infinite series solution, when $\lambda=0.1, b=1, k=1 / 3$, $t=0.001$, and $\mu=1$. Additionally, we can mention that the results shown in Figures 5 and 6 have the absolute error of the order of $10^{-12}-10^{-13}$ between the exact and approximated solutions, while the absolute error obtained in [36] turns to be greater, of the order of $10^{-5}-10^{-6}$.

Figures 7, 8, and 9 show the absolute error between the approximated solutions (17) and the analytical solutions (7) for the special cases of 5, 7, and 10 perturbative terms in the approximated solutions, when $\mu=0.97$ and $t=0.002$. We notice from these figures that the convergency of the solutions obtained by applying the HAM is reached by taking into account only a few number of perturbative terms in the solutions (17).

Similar results have been obtained for different values of the order of the fractional derivative $(\mu)$, as we can see in Table 1. In this table we have shown the absolute error for 
TABLE 1: Absolute error between the HAM solution $u_{\text {HAM }}(x, t)$ and the exact solution $u_{\text {exact }}(x, t)$ with $\mu=0.94$, for the 5th and 10th perturbation terms.

\begin{tabular}{|c|c|c|c|}
\hline$t$ & $x$ & $\left|u_{\text {exact }}(x, t)-u_{\mathrm{HAM}}(x, t)\right|(5$ th terms $)$ & $\left|u_{\text {exact }}(x, t)-u_{\text {HAM }}(x, t)\right|$ (10th terms) \\
\hline $1 / 1000$ & 0 & $5.59496 \times 10^{-10}$ & $5.59496 \times 10^{-10}$ \\
\hline $1 / 1000$ & $1 / 10$ & 0.000231565 & 0.000231565 \\
\hline $1 / 1000$ & $1 / 5$ & 0.000266312 & 0.000266312 \\
\hline $1 / 1000$ & $3 / 10$ & 0.000284713 & 0.000284713 \\
\hline $1 / 1000$ & $2 / 5$ & 0.000296001 & 0.000296001 \\
\hline $1 / 1000$ & $1 / 2$ & 0.000303069 & 0.000303069 \\
\hline $1 / 1000$ & $3 / 5$ & 0.00030722 & 0.00030722 \\
\hline $1 / 1000$ & $7 / 10$ & 0.000309167 & 0.000309167 \\
\hline $1 / 1000$ & $4 / 5$ & 0.000309357 & 0.000309357 \\
\hline $1 / 1000$ & $9 / 10$ & 0.000308099 & 0.000308099 \\
\hline $1 / 1000$ & 1 & 0.000305621 & 0.000305621 \\
\hline $1 / 500$ & 0 & $3.948657 \times 10^{-9}$ & $3.948657 \times 10^{-9}$ \\
\hline $1 / 500$ & $1 / 10$ & 0.000373782 & 0.000373782 \\
\hline $1 / 500$ & $1 / 5$ & 0.000441939 & 0.000441939 \\
\hline $1 / 500$ & $3 / 10$ & 0.00047875 & 0.00047875 \\
\hline $1 / 500$ & $2 / 5$ & 0.000501839 & 0.000501839 \\
\hline $1 / 500$ & $1 / 2$ & 0.00051681 & 0.00051681 \\
\hline $1 / 500$ & $3 / 5$ & 0.000526196 & 0.000526196 \\
\hline $1 / 500$ & $7 / 10$ & 0.000531384 & 0.000531384 \\
\hline $1 / 500$ & $4 / 5$ & 0.000533242 & 0.000533242 \\
\hline $1 / 500$ & $9 / 10$ & 0.000532363 & 0.000532363 \\
\hline $1 / 500$ & 1 & 0.000529188 & 0.000529188 \\
\hline $3 / 1000$ & 0 & $1.2381 \times 10^{-8}$ & $1.2381 \times 10^{-8}$ \\
\hline $3 / 1000$ & $1 / 10$ & 0.000486726 & 0.000486726 \\
\hline $3 / 1000$ & $1 / 5$ & 0.000586844 & 0.000586844 \\
\hline $3 / 1000$ & $3 / 10$ & 0.000641684 & 0.000641684 \\
\hline $3 / 1000$ & $2 / 5$ & 0.000676534 & 0.000676534 \\
\hline $3 / 1000$ & $1 / 2$ & 0.000699548 & 0.000699548 \\
\hline $3 / 1000$ & $3 / 5$ & 0.000714431 & 0.000714431 \\
\hline $3 / 1000$ & $7 / 10$ & 0.00072322 & 0.00072322 \\
\hline $3 / 1000$ & $4 / 5$ & 0.000727182 & 0.000727182 \\
\hline $3 / 1000$ & $9 / 10$ & 0.000727186 & 0.000727186 \\
\hline $3 / 1000$ & 1 & 0.000723875 & 0.000723875 \\
\hline
\end{tabular}

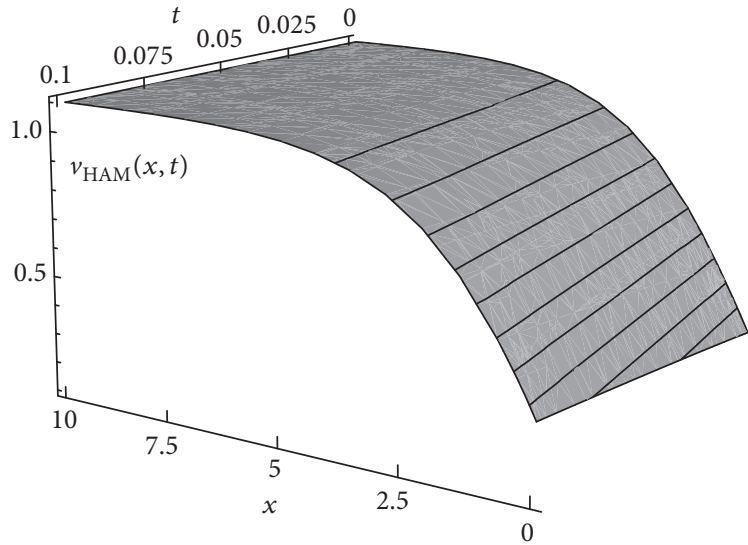

FIGURE 2: Approximated analytical solutions eq. (17) for $v(x, t)$, with $\lambda=0.1, b=1 ; k=1 / 3$, at $t=0.001$ and $\mu=0.97$.

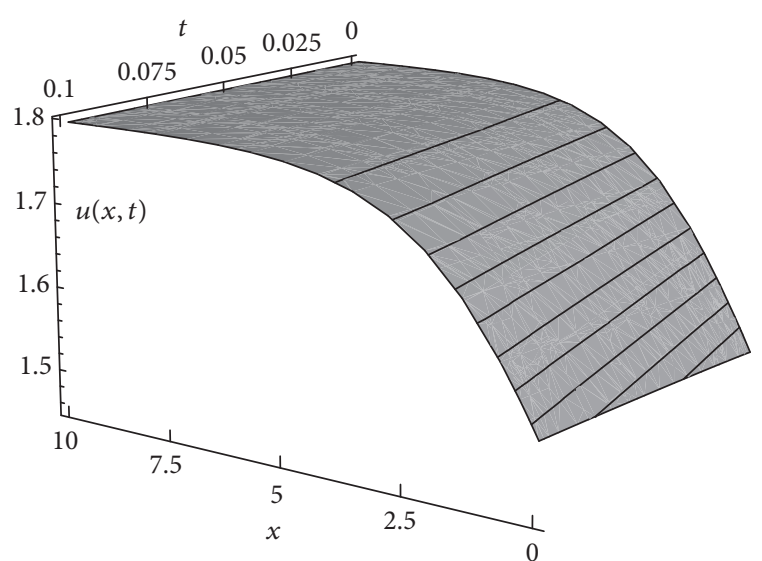

FIGURE 3: Exact solutions eq. (7) for $u(x, t)$, with $\lambda=0.1, b=1$; $k=1 / 3$, at $t=0.001$ and $\mu=0.97$. 


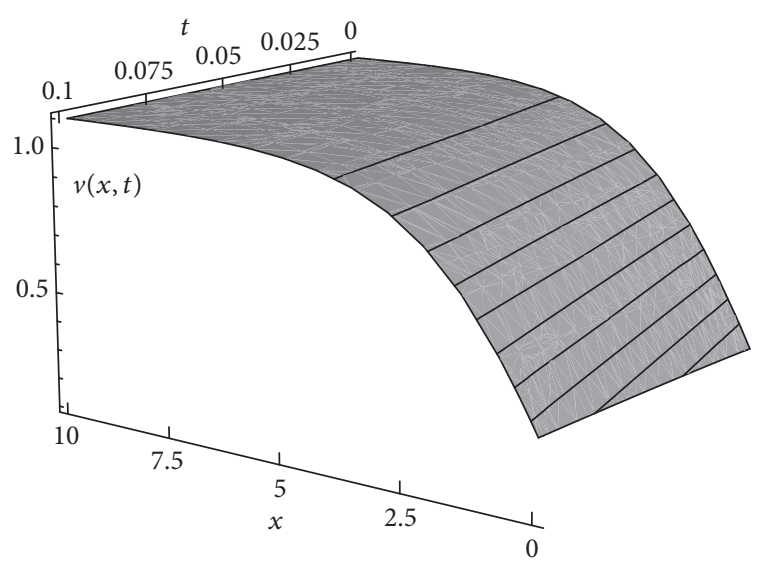

FIgURE 4: Exact solutions eq. (7) for $v(x, t)$, with $\lambda=0.1, b=1$; $k=1 / 3$, at $t=0.001$ and $\mu=0.97$.

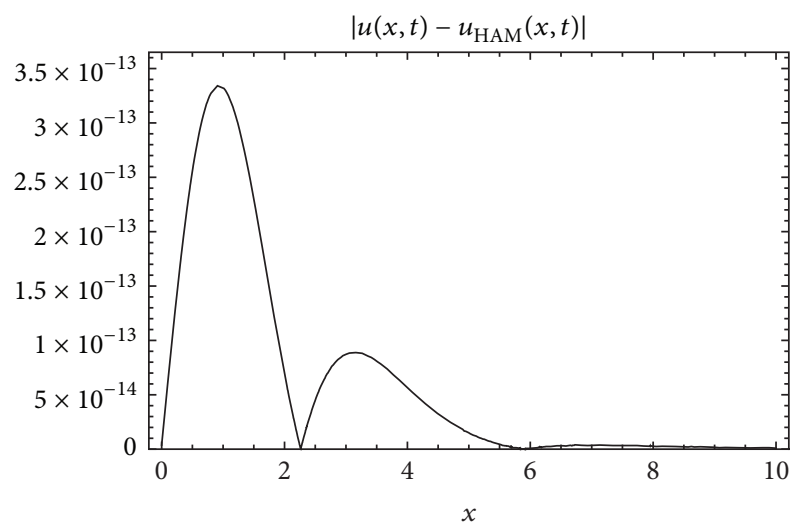

FIGURE 5: Absolute error between the approximated solution and the exact one for $u(x, t)$ at $t=0.001$, with $\mu=1$.

different values of $x$ and $t$, when $\mu=0.94$, for the special cases when 5 and 10 perturbative terms in the approximated solutions (17) were considered.

From Figures 7, 8, and 9 and Table 1 we observed the general behavior of the convergency of the solutions obtained when the HAM is applied to the $\mathrm{mKdV}$ equation. The accuracy of the solutions requires more terms in the perturbative series (17) as we go from the integer order $\mu=1$ to lower values. Also the accuracy of the solutions requires more terms in the perturbative series for greater values of the $t$ variable. We have obtained the same general behavior for the solution $v(x, t)$ of the $\mathrm{mKdV}$ equation.

\section{Conclusion}

Based on the HAM, in this paper, we obtain new approximated solutions of nonlinear coupled fractional mKdV equation; the solutions are given in a series form which converges rapidly. The methodologies presented become

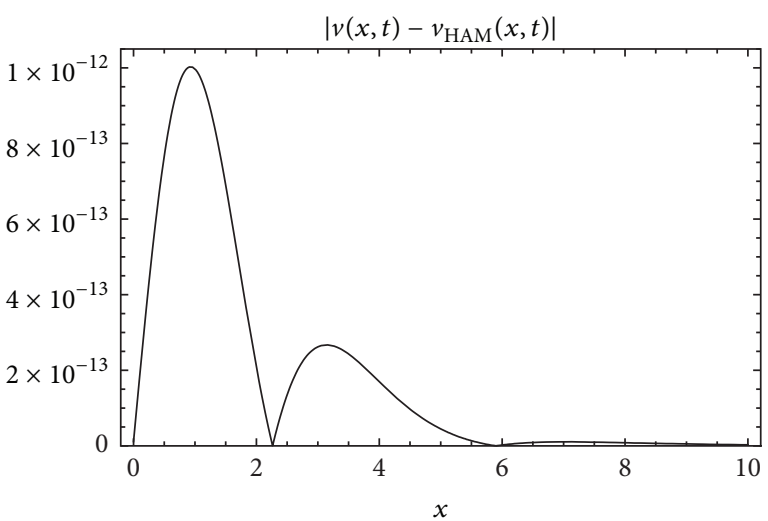

FIgURE 6: Absolute error between the approximated solution and the exact one for $v(x, t)$ at $t=0.001$, with $\mu=1$.

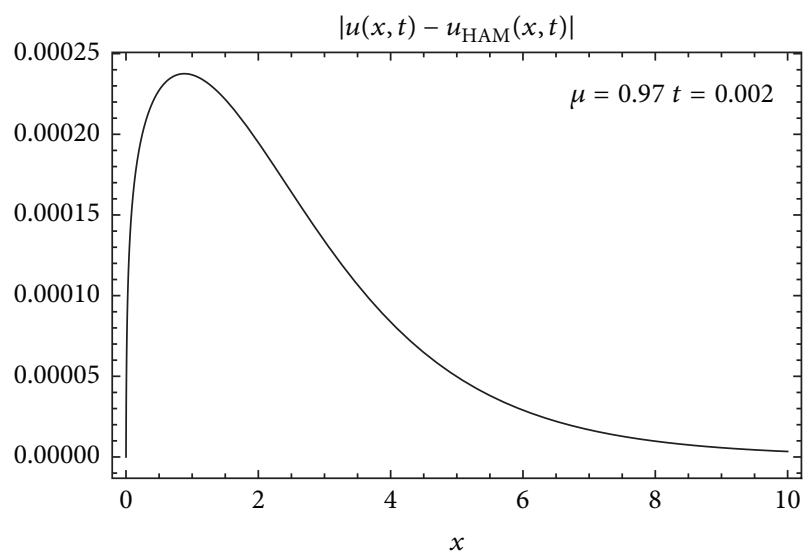

FIGURE 7: Absolute error between the approximated solution and the exact one for $u(x, t)$ at $t=0.002$, with $\mu=0.97$, when 5 perturbative terms in the solutions (17) are considered.

important mathematical tools, motivated by the potential useful for physics and engineers working in various areas of natural sciences. In this work Mathematica has been used for algebraic calculations.

To the best of our knowledge, the absolute error reported in Figures 5 and 6, between the exact solutions (7) and the analytical approximated solutions (17), for (1), has not been achieved before in the literature, when the HAM was applied to the $\mathrm{mKdV}$ equation.

\section{Competing Interests}

The authors declare no conflict of interests.

\section{Acknowledgments}

The authors gratefully acknowledge the Universidad Autónoma de la Ciudad de México for supporting and facilitating this research work. They would like to thank Mayra Martínez for the interesting discussions. José Francisco Gómez Aguilar 


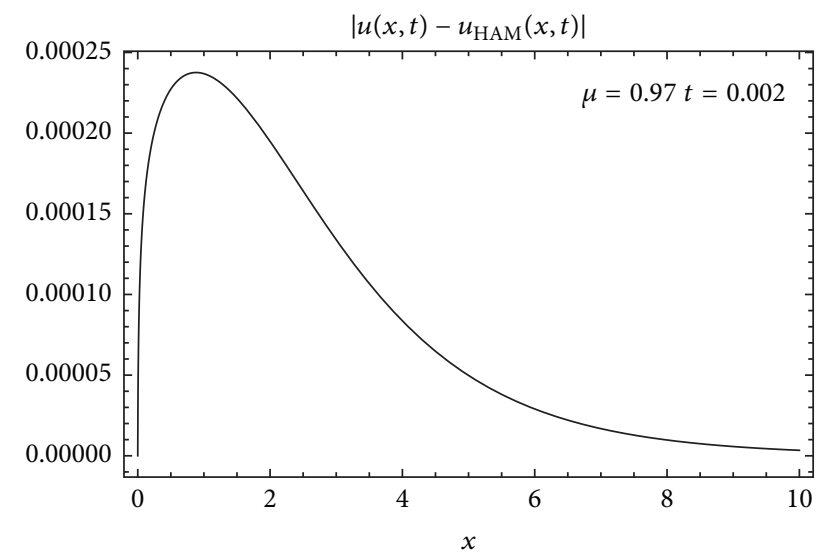

FIGURE 8: Absolute error between the approximated solution and the exact one for $u(x, t)$ at $t=0.002$, with $\mu=0.97$, when 7 perturbative terms in the solutions (17) are considered.

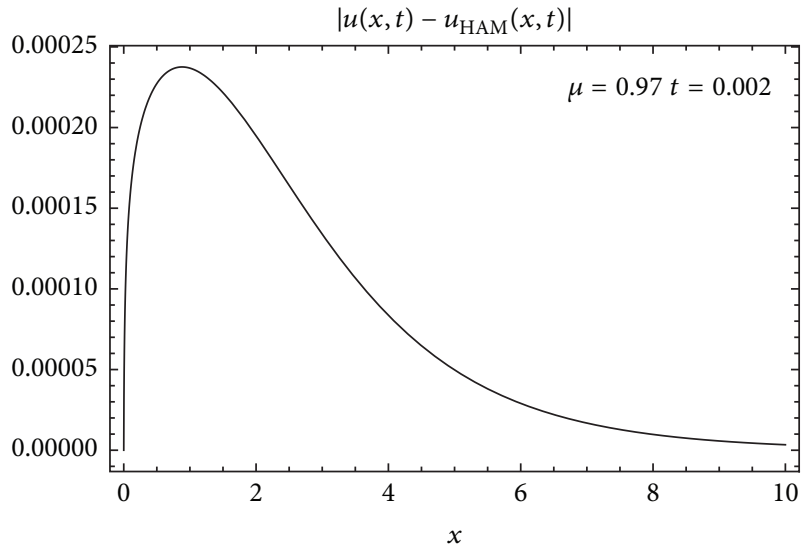

FIGURE 9: Absolute error between the approximated solution and the exact one for $u(x, t)$ at $t=0.002$, with $\mu=0.97$, when 10 perturbative terms in the solutions (17) are considered.

acknowledges the support provided by CONACYT: Cátedras CONACYT para Jovenes Investigadores 2014.

\section{References}

[1] R. Hilfer, "Experimental implications of Bochner-Levy-Riesz diffusion," Fractional Calculus and Applied Analysis, vol. 18, no. 2, pp. 333-341, 2015.

[2] A. W. Wharmby and R. L. Bagley, "The application of the fractional calculus model for dispersion and absorption in dielectrics I. Terahertz waves," International Journal of Engineering Science, vol. 93, pp. 1-12, 2015.

[3] D. Baleanu, K. Diethelm, E. Scalas, and J. J. Trujillo, Fractional Calculus Models and Numerical Methods, Complexity, Nonlinearity and Chaos, World Scientiffic, 2012.

[4] M. Eckert, M. Kupper, and S. Hohmann, "Functional fractional calculus for system identification of battery cells," AtAutomatisierungstechnik, vol. 62, no. 4, pp. 272-281, 2014.

[5] J. F. G. Gómez-Aguilar, T. C. Córdova-Fraga, J. E. EscalanteMartínez, C. Calderón-Ramón, and R. F. Escobar-Jiménez,
"Electrical circuits described by a fractional derivative with regular Kernel," Revista Mexicana de Física, vol. 62, pp. 144-154, 2016.

[6] J. F. G. Gómez-Aguilar, M. Miranda-Hernández, M. G. L. López-López, V. M. Alvarado-Martínez, and D. Baleanu, "Modeling and simulation of the fractional space-time diffusion equation," Communications in Nonlinear Science and Numerical Simulation, vol. 30, no. 1, pp. 115-127, 2016.

[7] P. A. Smyth and I. Green, "Fractional calculus model of articular cartilage based on experimental stress-relaxation," Mechanics of Time-Dependent Materials, vol. 19, no. 2, pp. 209-228, 2015.

[8] Ö. Güner and A. Bekir, "Exact solutions of some fractional differential equations arising in mathematical biology," International Journal of Biomathematics, vol. 8, no. 1, Article ID 1550003, pp. 1-17, 2015.

[9] J. F. Gómez-Aguilar, R. F. Escobar-Jiménez, V. H. OlivaresPeregrino, M. Benavides-Cruz, and C. Calderón-Ramón, "Nonlocal electrical diffusion equation," International Journal of Modern Physics C, vol. 27, no. 1, Article ID 1650007, pp. 1-12, 2016.

[10] D. T. Spasic, N. I. Kovincic, and D. V. Dankuc, "A new material identification pattern for the fractional Kelvin-Zener model describing biomaterials and human tissues," Communications in Nonlinear Science and Numerical Simulation, vol. 37, pp. 193199, 2016.

[11] G. C. Wu and D. Baleanu, "Reprint of: chaos synchronization of the discrete fractional logistic map," Signal Processing, vol. 107, pp. 444-447, 2015.

[12] B. Li and W. Xie, "Image denoising and enhancement based on adaptive fractional calculus of small probability strategy," Neurocomputing, vol. 175, pp. 704-714, 2016.

[13] Y. Dai, Y. Wei, Y. Hu, and Y. Wang, "Modulating function-based identification for fractional order systems," Neurocomputing, vol. 173, pp. 1959-1966, 2016.

[14] I. Tejado, D. Valério, E. Pérez, and N. Valério, "Fractional calculus in economic growth modelling: the Spanish and Portuguese cases," International Journal of Dynamics and Control, 2015.

[15] N. Das, R. Singh, A.-M. Wazwaz, and J. Kumar, "An algorithm based on the variational iteration technique for the Bratutype and the Lane-Emden problems," Journal of Mathematical Chemistry, vol. 54, no. 2, pp. 527-551, 2016.

[16] P. R. Mistry and V. H. Pradhan, "Approximate analytical solution of non-linear equation in one dimensional instability phenomenon in homogeneous porous media in horizontal direction by variational iteration method," Procedia Engineering, vol. 127, pp. 970-977, 2015.

[17] J.-S. Duan, R. Rach, and A. M. Wazwaz, "Higher order numeric solutions of the Lane-Emden-type equations derived from the multi-stage modified Adomian decomposition method," International Journal of Computer Mathematics, 2015.

[18] J. S. Duan, R. Rach, and A. M. Wazwaz, "Steady-state concentrations of carbon dioxide absorbed into phenyl glycidyl ether solutions by the Adomian decomposition method," Journal of Mathematical Chemistry, vol. 53, no. 4, pp. 1054-1067, 2015.

[19] A. Ghorbani, "Beyond Adomain's polynomials: He's polynomials," Chaos, Solitons \& Fractals, vol. 39, pp. 1486-1492, 2009.

[20] L. Lu, J. Duan, and L. Fan, "Solution of the magnetohydrodynamics jeffery-hamel flow equations by the modified adomian decomposition method," Advances in Applied Mathematics and Mechanics, vol. 7, no. 5, pp. 675-686, 2015. 
[21] H. C. Ma, D. D. Yao, and X. F. Peng, "Exact solutions of nonlinear fractional partial differential equations by fractional subequation method," Thermal Science, vol. 19, no. 4, pp. 1239-1244, 2015.

[22] S. Guo and L. Mei, "Exact solutions of space-time fractional variant Boussinesq equations," Advanced Science Letters, vol. 10, no. 1, pp. 700-702, 2012.

[23] S. T. Mohyud-Din, T. Nawaz, E. Azhar, and M. A. Akbar, "Fractional sub-equation method to space-time fractional CalogeroDegasperis and potential Kadomtsev-Petviashvili equations," Journal of Taibah University For Science, 2015.

[24] H. Jafari, M. Ghorbani, and S. Ghasempour, "A note on 'exact solutions for nonlinear integral equations by a modified homotopy perturbation method"' New Trends in Mathematical Sciences, vol. 1, no. 2, pp. 22-26, 2013.

[25] U. F. Nino, H. Vázquez-Leal, Y. Khan, D. Pereyra-Díaz, A. Pérez-Sesma, and A. Díaz-Sánchez, "Modified nonlinearities distribution Homotopy Perturbation method as a tool to find power series solutions to ordinary differential equations," Nova Scientia, vol. 6, no. 12, pp. 13-38, 2014.

[26] E. Cuce and P. M. Cuce, "A successful application of homotopy perturbation method for efficiency and effectiveness assessment of longitudinal porous fins," Energy Conversion and Management, vol. 93, pp. 92-99, 2015.

[27] K. Sayevand and H. Jafari, "On systems of nonlinear equations: some modified iteration formulas by the homotopy perturbation method with accelerated fourth- and fifth-order convergence," Applied Mathematical Modelling, vol. 40, no. 2, pp. 1467-1476, 2016.

[28] S. Liao, Homotopy Analysis Method in Nonlinear Differential Equations, Springer, Berlin, Germany, 2012.

[29] J. Rahimi, M. R. Esboee, D. D. Ganji, I. Rahimi-Petrodi, and R. Mohammadyari, "Implementation of Homotopy Analysis Method on circular permeable slider containing of incompressible Newtonian fluid," Boletim da Sociedade Paranaense de Matemática, vol. 34, no. 1, pp. 21-31, 2016.

[30] J. F. Gómez-Aguilar, L. Torres, H. Yépez-Martínez, D. Baleanu, J. M. Reyes, and I. O. Sosa, "Fractional Liénard type model of a pipeline within the fractional derivative without singular kernel," Advances in Difference Equations, vol. 2016, no. 1, article 173, pp. 1-13, 2016.

[31] V. F. Morales-Delgado, J. F. Gómez-Aguilar, H. Yépez-Martínez, D. Baleanu, R. F. Escobar-Jiménez, and V. H. OlivaresPeregrino, "Laplace homotopy analysis method for solving linear partial differential equations using a fractional derivative with and without kernel singular," Advances in Difference Equations, vol. 2016, no. 1, article 164, pp. 1-6, 2016.

[32] H. Jafari and S. Seifi, "Solving a system of nonlinear fractional partial differential equations using homotopy analysis method," Communications in Nonlinear Science and Numerical Simulation, vol. 14, no. 5, pp. 1962-1969, 2009.

[33] M. Caputo, "Linear models of dissipation whose Q is almost frequency independent-part II," Geophysical Journal International, vol. 13, no. 5, pp. 529-539, 1967.

[34] M. Garshasbi and F. Momeni, "Numerical solution of HirotaSatsuma coupled MKdV equation with quantic B-Spline collocation method," Journal of Computer Science \& Computational Mathematics, vol. 1, no. 3, pp. 13-18, 2011.

[35] S. Effati, H. S. Nik, and R. Buzhabadi, "Solving famous nonlinear coupled equations with parameters derivative by homotopy analysis method," International Journal of Differential Equations, vol. 2011, Article ID 545607, 15 pages, 2011.
[36] Y. Chen and H. An, "Homotopy perturbation method for a type of nonlinear coupled equations with parameters derivative," Applied Mathematics and Computation, vol. 204, no. 2, pp. 764$772,2008$.

[37] J. Liu and H. Li, "Approximate analytic solutions of timefractional Hirota-Satsuma coupled KdV equation and coupled MKdV equation," Abstract and Applied Analysis, vol. 2013, Article ID 561980, 2013.

[38] E. G. Fan, "Soliton solutions for a generalized Hirota-Satsuma coupled KdV equation and a coupled MKdV equation," Physics Letters, Section A: General, Atomic and Solid State Physics, vol. 282, no. 1-2, pp. 18-22, 2001.

[39] B. J. West, M. Bologna, and P. Grigolini, Physics of Fractal Operators, Springer, New York, NY, USA, 2003.

[40] I. Podlubny, Fractional Differential Equations, Academic Press, San Diego, Calif, USA, 1999.

[41] H. Yépez-Martínez, J. M. Reyes, and I. O. Sosa, "Fractional sub-equation method and analytical solutions to the HirotaSatsuma coupled KdV equation and coupled mKdV equation," British Journal of Mathematics \& Computer Science, vol. 4, no. 4, pp. 572-589, 2014.

[42] J. Zhao, B. Tang, S. Kumar, and Y. Hou, "The extended fractional subequation method for nonlinear fractional differential equations," Mathematical Problems in Engineering, vol. 2012, Article ID 924956, 11 pages, 2012.

[43] L. N. Song and H. Q. Zhang, "Solving the fractional BBMBurgers equation using the homotopy analysis method," Chaos, Solitons \& Fractals, vol. 40, no. 4, pp. 1616-1622, 2009.

[44] K. A. Gepreel and M. S. Mohamed, "Analytical approximate solution for nonlinear space-time fractional Klein-gordon equation," Chinese Physics B, vol. 22, no. 1, Article ID 010201, 2013.

[45] K. M. Hemida, K. A. Gepreel, and M. S. Mohamed, "Analytical approximate solution to the time-space nonlinear partial fractional differential equations," International Journal of Pure and Applied Mathematics, vol. 78, no. 2, pp. 233-243, 2012. 


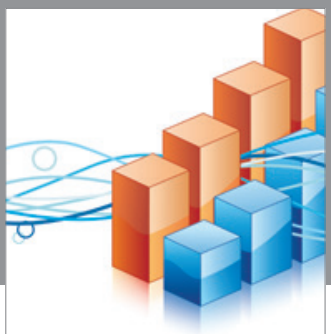

Advances in

Operations Research

vatem alat4

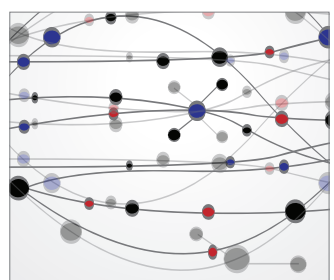

\section{The Scientific} World Journal
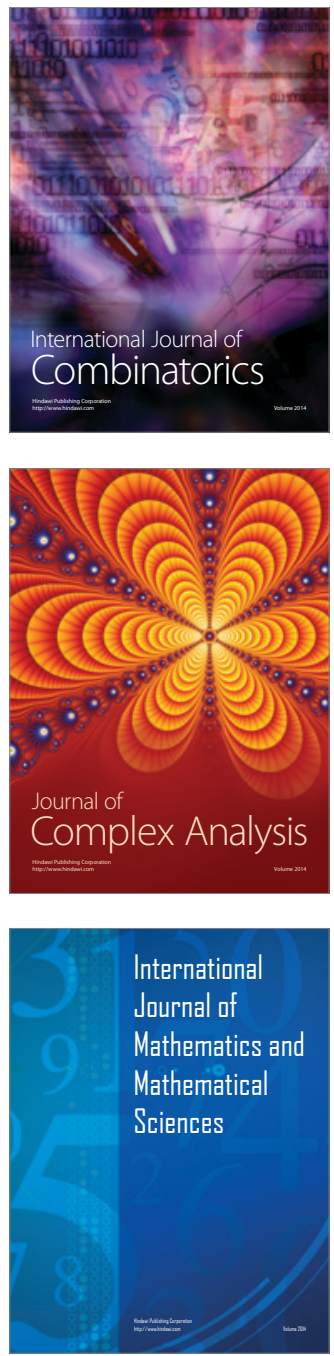
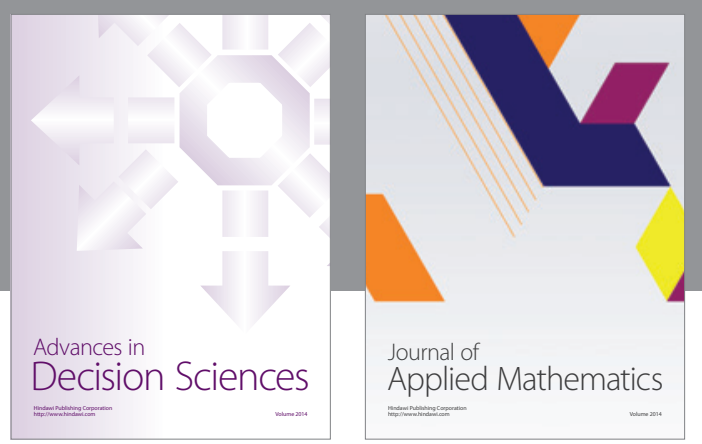

Algebra

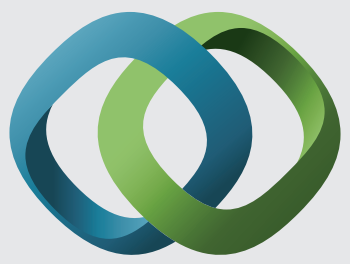

\section{Hindawi}

Submit your manuscripts at

http://www.hindawi.com
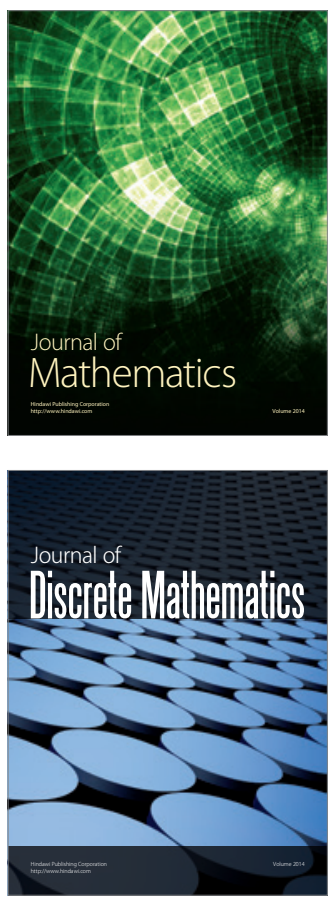

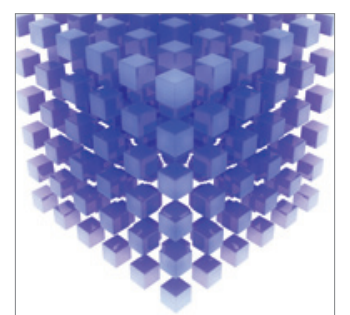

Mathematical Problems in Engineering
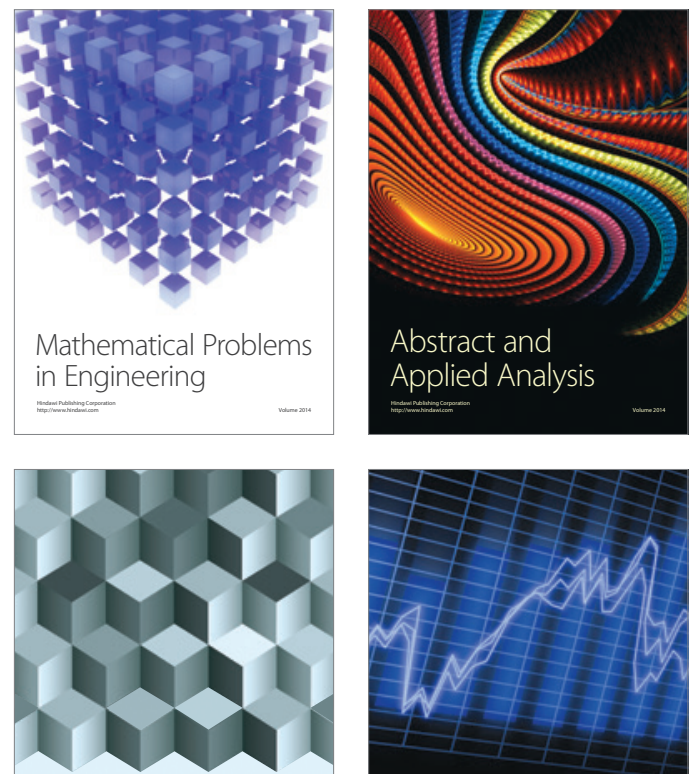

Journal of

Function Spaces

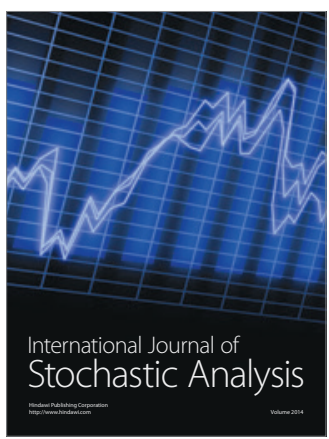

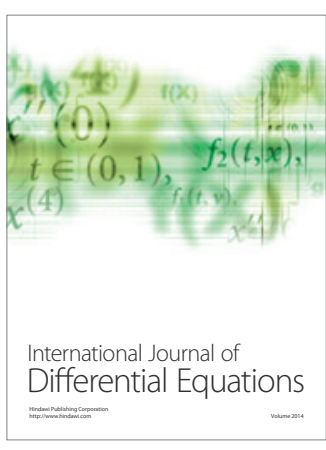
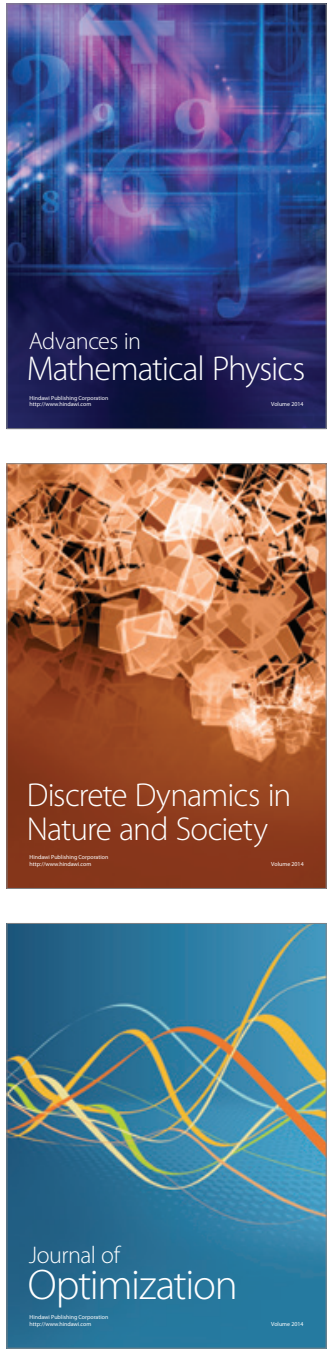